\title{
Regularity Comparativism about Mass in Newtonian Gravity*†
}

\author{
Niels C.M. Martens ${ }^{\ddagger \S}$
}

\begin{abstract}
Comparativism - the view that mass ratios are not grounded in absolute massesfaces a challenge by Baker which suggests that absolute masses are empirically meaningful. Regularity comparativism uses a liberalised version of the Mill-Ramsey-Lewis Best Systems Account to have both the Laws of Newtonian Gravity and the absolute mass scale supervene on a comparativist Humean mosaic as a package deal. I discuss three objections to this view, and conclude that it is untenable. The most severe problem is that once we have reduced away the absolute masses, there is nothing that stops us from also reducing the mass ratios.
\end{abstract}

\footnotetext{
${ }^{*}$ I would like to thank Casey McCoy, Tushar Menon and Oliver Pooley for useful discussions and comments on earlier drafts of this paper. I am grateful for questions and comments from the audiences at the following 2016 seminars and conferences: "Philosophy of Physics Graduate Lunch Seminar" and "Philosophy of Physics Research Seminar" at the University of Oxford, "Society for the Metaphysics of Science Annual Conference" at the University of Geneva, and "PSA2016" in Atlanta. This material is based upon work supported by the Arts \& Humanities Research Council of the UK, a Scatcherd European Scholarship, and in part by the DFG Research Unit "The Epistemology of the Large Hadron Collider" (grant FOR 2063).

${ }^{\dagger}$ Forthcoming in Philosophy of Science 84(5), 2017.

$¥$ Magdalen College \& Dept. of Philosophy, University of Oxford, United Kingdom

$\S$ Institute for Theoretical Particle Physics and Cosmology, RWTH Aachen University, Germany, martens@physik.rwth-aachen.de
} 
Copyright Philosophy of Science 2017

Preprint (not copyedited or formatted)

Please use DOI when citing or quoting

\section{Introduction}

In response to Newton's bucket - i.e. the argument from inertial effects, against relationalism about space, which purports to show the empirical meaningfulness of inertial frames - Van Fraassen (1970) has pointed out that it is not the structure of absolute space that is required to privilege certain frames, but merely the truth of Newton's laws in those frames. Huggett (2006) uses this insight to formulate a version of relationalism that 'gets inertial frames for free'. Regularity Relationalism employs the framework of Humean Supervenience - in particular the Mill-Ramsey-Lewis Best Systems Account-to have both the inertial frames and the laws supervene as a package deal on a relational Humean mosaic.

Comparativism about mass (Dasgupta 2013; Baker 2013; Martens 2017) - the view that mass ratios are not grounded in absolute masses - faces a challenge analogous to Newton's bucket. If we consider a two-particle world governed by the laws of Newtonian Gravity, the comparativist initial conditions (distance, velocity and mass ratios) will be insufficient to provide a deterministic evolution of the system, as the escape velocity in-

equality $\left(v_{e}>\sqrt{\frac{2 G m}{r}}\right)$ depends on the absolute masses over and beyond the mass ratios (Baker 2013). This 'comparativist's bucket' suggests that the absolute mass scale is empirically meaningful (Martens 2017). An obvious comparativist response seems to be a regularity version of comparativism that attempts to ground in a comparativist Humean mosaic both the absolute mass scale and the laws of Newtonian Gravity as a package deal.

After outlining the framework of Humean Supervenience and in particular the MillRamsey-Lewis Best Systems Account in Section 2, I discuss regularity relationalism (Section 3) and regularity comparativism (Section 4). The remaining three sections discuss three arguments against regularity comparativism in Newtonian Gravity, leading to the conclusion that this view is untenable.

\section{Empiricism about Laws of Nature}

Perhaps the most popular incarnation of empiricism about laws of nature goes under the name of Humean Supervenience. Earman already considered whether "laws are parasitic on occurrent facts" in 1984, but the most well-known formulation of the view stems from Lewis in 1986:

Humean supervenience is named in honor of the greater [sic] denier of necessary connections. It is the doctrine that all there is to the world is a vast mosaic of local matters of fact, just one little thing and then another. [...] We have geometry: a system of external relations of spatio-temporal distances between points. Maybe points of spacetime itself, maybe pointsized bits of matter 
Copyright Philosophy of Science 2017

Preprint (not copyedited or formatted)

Please use DOI when citing or quoting

or aether fields, maybe both. And at those points we have local qualities: perfectly natural instrinsic properties which require nothing bigger than a point at which to be instantiated. For short: we have an arrangement of qualities. And that is all. All else supervenes on that. (Lewis 1986, ix-x)

The name 'Humean Supervenience' suggests that this view in fact goes much further back than 1986, but we will question below whether Lewis' motivations and justifications were indeed the same as Hume's.

As Maudlin points out, Lewis' thesis of Humean Supervenience in fact comprises two logically independent theses (2007b).

The first states that:

Separability: "The complete physical state of the world ... supervenes on ... the intrinsic physical state of each spacetime point (or each pointlike object) and the spatiotemporal relations between those points." (2007b, 51)

Or, as Maudlin informally glosses it, all fundamental properties are intrinsic properties, except for spatio-temporal relations, which are the only fundamental external properties (i.e. relations).

In the context of this paper it will prove useful to note that Separability - at least according to the informal gloss - seems to comprise two additional theses. Firstly, it is committed to Strong Absolutism about all non-spatio-temporal quantities ${ }^{1}$ such as mass and electric charge, i.e. both (Weak) Absolutism - the view that absolute masses ground mass ratios (Dasgupta 2013; Martens 2017) - and Quantity Primitivism (QP). The latter is the view that these absolute quantities are fundamental. Secondly, it is committed to 4D-fundamentalism: the view that the four-dimensional spatio-temporal relations are fundamental (as opposed to for instance a $3 \mathrm{~N}$-dimensional configuration space) ${ }^{2}$.

It is not directly clear that the formal definition of Separability does indeed commit to either QP or 4D-fundamentalism, because both theses only follow from twice sneaking in the notion of fundamentality into the informal gloss. We will consider using this wiggle room below. One could perhaps argue though that Lewis' original quote is most naturally interpreted by adding those fundamentality qualifiers.

What is clear is that the formal definition commits one to absolutism about all nonspatio-temporal quantities. As it stands, Humean Supervenience is thus clearly not usable by comparativists. Below we will discuss the option of liberalising Humean Supervenience, in order to make it suitable for comparativists.

Besides Separability, Humean Supervenience comprises Supervenience ${ }^{3}$ :

\footnotetext{
${ }^{1}$ In my DPhil thesis (2017) I argue that these views are to be understood in terms of what I call 'magnitudes', rather than quantities. Here the difference is not too important.

${ }^{2}$ Here I use terminology that is similar to Chen's (2016).

'Physical Statism' in Maudlin's terminology.
} 
Copyright Philosophy of Science 2017

Preprint (not copyedited or formatted)

Please use DOI when citing or quoting

Supervenience: "All facts about a world, including modal and nomological facts, are determined by its [complete] physical state." (Maudlin 2007b, 51)

Separability of course requires the physical state referred to in Supervenience to be separable, but Supervenience by itself does not do so as it is a logically independent thesis. How each thesis is motivated, and whether they can be motivated independently is an issue that we will return to below.

Within this broad framework of Humean Supervenience, the exact manner in which the nomological facts supervene on the separable physical state (i.e. the four-dimensional Humean mosaic) is usually explicated via the Mill-Ramsey-Lewis Best Systems Account (MRL) (Earman 1984; Lewis 1973). According to this approach, laws of nature are generalisations which are axioms or theorems of the 'best' axiomatisations of the Humean mosaic. What makes an axiomatisation the best axiomatisation is an optimalisation of two virtues that pull in opposite directions: simplicity and strength. The desideratum of strength, or informativeness, is often quantified by the 'amount' of possible worlds it rules out; simplicity is often cashed out syntactically.

\section{Regularity Relationalism}

Huggett (2006) defends a version of relationalism about space, regularity relationalism, which makes crucial use of the MRL approach. This version is supposed to deal with, inter alia, the problem posed by Newton's bucket, i.e. inertial effects. The substantivalist posits fundamental absolute space to provide the inertial frames in which Newton's laws hold and with respect to which the absolute acceleration is determined that underlies Newton's bucket. Huggett however builds upon the key insight by Van Fraassen (1970) that it is not the structure of absolute space that privileges certain frames, but merely the truth of Newton's laws in those frames. Thus, if we consider all the possible reference frames that are naturally adapted (to be specified in more detail below) to the spatiotemporal relations of the Humean mosaic, for some of these frames the laws that are the best axiomatisations will be Newton's laws, whereas the other frames will have best axiomatisations comprising laws that include additional correction terms. The plausible claim is then that the former set of reference frames provide the simplest (and strongest) axiomatisation overall. It is only those frames in which Newton's laws are true; it is merely this fact that privileges those frames, no further ontological implications can be derived from their privileged status. Thus, both the inertial frames and Newton's laws supervene as a package deal; the inertial frames come for free.

Now in slightly more detail. Huggett's sparse mosaic consists merely of the Leibnizian spatial relations of the particles over time, and their fundamental intrinsic properties such 
Copyright Philosophy of Science 2017

Preprint (not copyedited or formatted)

Please use DOI when citing or quoting

as mass and charge. Call this the relational state, or the Leibnizian-Humean mosaic. It does not include other geometric relations, and in particular not an affine structure.

We then consider the set of what we will call ontological coordinate frames. As a first pass, we might choose them to be the set of all adapted frames of all the bodies in the world. A frame is adapted to a reference body if that body is at rest at the origin of that frame. But, since in a world governed by Newtonian Gravity we would generally not expect there to be any inertial bodies - since gravity cannot be shielded off - this set of adapted frames would not include any inertial adapted frames. Instead we choose as our set of ontological frames the set of adapted frames plus all frames related to those adapted frames by arbitrary continuous spatially rigid transformations ${ }^{4}$.

In the final step we then axiomatise the Leibnizian-Humean mosaic separately for each of these ontological frames. This will, Huggett claims, result in a privileged set of pairs of Best System Frames and laws written in terms of those coordinates which are the simplest (and strongest). These laws are Newton's laws, and the frames are the inertial frames.

\section{Regularity Comparativism}

Regularity relationalism was inter alia a response to Newton's bucket, which demonstrated the empirical meaningfulness of a standard of inertia. The comparativist's bucket, analogously, demonstrates the empirical meaningfulness of an absolute mass scale. An obvious move for the comparativist is to use the regularity approach: getting the absolute mass scale for free by having it supervene on (or grounding it in) a mosaic of fundamental mass relations (as a package deal together with the laws of Newtonian Gravity).

Huggett's insight, building upon Van Fraassen's insight, was that if we quantify over the best axiomatisations of the Leibnizian-Humean mosaic as coordinatised by different ontological frames, the inertial subset of those frames will drop out, since only in those frames do the laws come out in their simplest Newtonian form. This easily translates to the case of comparativism. That one absolute mass scale is privileged does not imply that we should attribute it fundamental ontological status (relative to the mass ratios); it only means - as the claim goes - that when we quantify over the best axiomatisations of the Humean mosaic as 'coordinatised' by different absolute mass scales, the simple Newtonian laws will be true for only one of those choices of scale.

Again in slightly more detail. Our Comparativist Humean mosaic consists of the geometric structure (either merely the Leibnizian relations, or more structure) of all the bodies over time, plus fundamental mass ratios between all ${ }^{5}$ bodies, plus their fundamental

\footnotetext{
${ }^{4}$ Pooley chooses these frames ab initio $(2013, \S 3.1)$.

${ }^{5}$ In the case of Chain Comparativism (Martens 2017, Ch.5), there will only be a single chain of fundamental relations, not a complete graph/web.
} 
Copyright Philosophy of Science 2017

Preprint (not copyedited or formatted)

Please use DOI when citing or quoting

intrinsic properties such as charge.

Clearly this constitutes a further liberalisation of the standard form of Humean Supervenience, since both the formal and informal definition of Separability presupposed absolutism. Moreover, the informal definition comprised Quantity Primitivism about absolute masses, whereas, although absolute masses still 'exist' in regularity comparativism, they are not fundamental ${ }^{6}$. We will discuss below whether this is a problem, and if so how it might be solved. For now we will just go with it.

The analogue of the sets of ontological coordinates is the sets of distributions of absolute mass quantities that are compatible with the fundamental mass ratios. Since a choice of absolute mass for one particle fixes all the other absolute masses via the mass ratios, this set of ontological mass coordinates can be parameterised by the absolute mass quantity of one specific body.

For each value of the absolute mass scale parameter we consider the best axiomatisation of the mosaic so 'coordinatised'. The claim is then that only for the single correct value of the parameter the best axiomatisation comprises the laws of Newtonian Gravity, and that these are the best laws overall.

\section{$5 \quad$ A disanalogy with regularity relationalism?}

In the remaining three sections I will discuss three objections to regularity comparativism about mass in Newtonian Gravity. The first can be avoided; the second raises more serious problems; the third is fatal.

One might concede that the regularity protocol provides the inertial frames, but nevertheless fails to provide the absolute mass scale, by arguing that the mass and space cases are disanalogous.

The regularity approach claims that only for a subset of the ontological 'coordinates' the laws are best, and those best laws are the laws of Newtonian Gravity. In other words: $L\left(c_{o}\right)=L_{N G}\left(c_{o}\right)+L_{c o r r}\left(c_{o}\right)$, that is in general there will be correction terms (i.e. fictional forces in the space case). Only for the Best System Coordinates (i.e. the inertial frames in the space case) will the correction terms go to zero: $L\left(c_{b s}\right)=L_{N G}\left(c_{b s}\right)$. Finally, and crucially, there is no alternative way of writing $L_{N G}\left(c_{o}\right)+L_{c o r r}\left(c_{o}\right)$ (for coordinates that are not part of the best system) that is as simple as $L_{N G}\left(c_{b s}\right)$.

Now, this final claim seems plausible in the case of regularity relationalism. But, the argument goes, it is much less plausible in the case of comparativism. It is true that if we do not change the units of mass, position, Newton's constant and the accelerations involved, choosing the wrong absolute mass scale will mean that $L_{N G}\left(c_{o}\right)$ maps those masses to the wrong numerical values of the accelerations (i.e. the data that we are trying

\footnotetext{
${ }^{6}$ Nor intrinsic (Martens 2017).
} 
Copyright Philosophy of Science 2017

Preprint (not copyedited or formatted)

Please use DOI when citing or quoting

to account for). This would imply that a correctional term is required. But, the argument goes, what if, whenever we choose the 'incorrect' absolute mass scale, we just adjust the numerical value of Newton's constant accordingly. $L_{N G}\left(c_{o}(G)\right)+L_{\text {corr }}\left(c_{o}\right)$ can always be rewritten as $L_{N G}\left(c_{o}\left(G^{\prime}\right)\right)$. Thus, for each choice of mass scale we could get an equally simple law; the only difference is the numerical value of $\mathrm{G}$ in each of these laws. In other words, the regularity approach fails in its main aim: picking out a unique absolute mass scale.

The correct response ${ }^{7}$ is that this aim is too strict. Even in the space case, the regularity protocol picks out not one frame but a set of inertial frames, related by the Galilean Symmetry of the theory (or translations). In the mass case, only $G \cdot m$ is empirically accessible, not the absolute mass separately (cf. the escape velocity inequality). Scaling $m$ and $G$ such that $G \cdot m$ remains constant is a symmetry of the theory. What the comparativist's bucket really demonstrates is the empirical meaningfulness of $G \cdot m$. The correct aim is then to pick out a unique $G \cdot m$, which of course implies that we expect the regularity protocol to pick out a specific (infinitely large) set of pairs of $G$ 's and $m$ 's. The regularity protocol does seem to succeed in this aim.

\section{Separability}

As pointed out earlier, regularity comparativism is blatantly contradicting one of the two theses comprising Humean Supervenience, the thesis of Separability, which presupposes absolutism about all non-spatio-temporal quantities. Two options immediately spring to mind: either liberalising or generalising the definition of Separability such that (regularity) comparativism does in fact satisfy it, or giving up Separability altogether and arguing that Supervenience can be used by itself to carry out the regularity protocol.

The latter option might seem best. The crux of the regularity approach is to have the inertial frames or the absolute mass scale supervene on some appropriate mosaic. The separability of that mosaic does not seem to play much of a role. Nevertheless, the Humeans have had to deal with one notorious charge of non-separability before, and in that case they were very reluctant to stray too far from the Separability condition. This is the case of entanglement in quantum mechanics. The details of this case are neatly rehearsed by, for instance, Maudlin (2007a), and need not detain us here. What is relevant here is that the Humeans accept that entangled quantum states are non-separable in the strict sense, but insist on defending some generalised version of Separability rather than just giving up any sort of separability condition altogether. Albert (1996) notes that the wave function lives in $3 N$-dimensional configuration space (with $N$ the number of particles in the

\footnotetext{
${ }^{7}$ I would like to thank Chris Wüthrich for insisting that this response has more to it than I originally thought.
} 
Copyright Philosophy of Science 2017

Preprint (not copyedited or formatted)

Please use DOI when citing or quoting

universe), and the wavefunction does specify intrinsic values (namely two, the amplitude and the phase) for each point of that higher-dimensional space. It is thus suggested that we should give up on 4D-fundamentalism and take configuration space to be the fundamental arena of physics. (Of course one then needs to tell a story of how this is consistent with our manifest image of the world around us being four-dimensional. Albert tells such a story, but we need not dwell on it here.) We could then define an analogue of Separability by quantifying instead over the points of that configuration space, and we may naturally want to call this new condition Generalised Separability.

Enter Shakespeare. "What's in a name?" Why should we care whether this new condition is satisified or not? Simply because its name is similar to a previous condition we cared about? In fact, why did we ever care about Separability? Albert's implicit motivation seems to be that the classical world, that is "familiar macroscopic objects' under so-called 'familiar macroscopic circumstances'" (1996, 282), is manifestly separable (assuming absolutism of course). Maudlin finds similar motivations in Lewis and Einstein. But the argument that new physics should be separable because physics so far has been separable is an inductive argument. We would expect Humeans, of all people, to be the last to endorse such an invalid argument. Even if this motivation were valid, it is not clear why this in any way would motivate Generalised Separability. This motivation is clearly about the 4D manifestion of the classical world. Moreover, even if a separate motivation for General Separability were to be provided (for instance realism about quantum mechanics, as Albert suggests), General Separability never did replace Separability. As hinted at earlier, the formal definition of Separability in consistent with quantification over emergent spacetime points. In other words, even if the wave function is separable in the generalised sense in configuration space, it would still be non-separable at the level of the 4D spacetime that has to emerge somehow from the configuration space.

We have arrived back at the suggestion of just leaving Separability behind, and sticking only with Supervenience (as Dasgupta does [2013]). From the standpoint of the regularity approach, this is all that we need. However, even though Supervenience is logically independent from Separability, we need to make sure that we can still motivate Supervenience once Separability is given up. We need to make sure that they did not come as a package deal. Why might we be motivated to hold Supervenience?

Let us recall the first sentence of the quote from Lewis: "Humean supervenience is named in honor of the greater [sic] denier of necessary connections." (1986, ix). Hume (1777) is famous for arguing that necessary connections between two events (the first of which we call the cause and the second of which we call the effect) are merely ideas of the mind, but not 'things' that can be observed. All that can be observed is constant conjugation of pairs of events. Humean empiricists therefore urge us to purge necessary connections (between what we call causes and effects), that is causation, from our metaphysics. Insofar as the notions of causation and laws are related, this form of empiricism 
Copyright Philosophy of Science 2017

Preprint (not copyedited or formatted)

Please use DOI when citing or quoting

also gets rid of laws as fundamental concepts.

But if it is an empiricist dislike for necessary connections that motivates Supervenience, then Humean Supervenience comes as a package deal. This type of empiricist will also be committed to a separable mosaic. As Dewar puts it: "Prima facie, the kind of world that violates Separability is one in which there are necessary connections between distinct existents: that is, in which there are fundamental and irreducible relations between pointlike things" $(2015,15)$ (italics in the original). Entangled states are such that, say, if a measurement on one of the entangled particles results in 'up', the measurement of the other one will necessarily result in 'down'. Note that 'necessarily' is here not to be read (just) as an inter-world notion, but rather an intra-world notion in the same sense as when Hume argues against one event (the cause) necessitating another later event in the same world (the effect). If there are two distinct physical systems consisting of massive particles, the behaviour of one will necessitate the behaviour of the other given the inter-system mass ratios. This does not detract from the mass ratios being totally contingent in the sense that they can differ between worlds/mosaics. The Humean might retort that on their framework the mass ratios and the trajectories are completely independently specifiable. In particular, we are free to have the second system behave differently from the way one would expect given the inter-system mass ratios. However, not only would this result in a world that is nomologically impossible according to Newtonian Gravity, it is also to admit that mass is in no way related to anything that is observable and it is therefore redundant. As will be discussed below, purging any notion of mass from the mosaic (since the trajectories do all the work by themselves) leads to what I believe to be the most defensible Humean position - regularity eliminativism - but is fatal for regularity comparativism. To sum up, if this dislike for necessary connections is the only motivation for Humean Supervenience, then this motivates both Supervenience and Separability. Separability cannot be given up without the whole thesis collapsing. A combination of comparativism and Humeanism is doomed from the start.

We have yet one other motivation to consider though. Huggett himself mentions that ontological parsimony (i.e. Ockham's razor) is the best justification for Humeanism: "The best argument for MRL is that it is metaphysically parsimonious and faces no knockdown objection" (2006, fn.3). Whether a Separable physical state is more ontologically parsimonious than a non-separable state is discussed elsewhere (Martens 2017, Ch.5). Here this is not immensely relevant. In contrast to the motivation from Humean Empiricism, where the dislike for necessary connections was a black-and-white matter which implied both Separability and Supervenience, ontological parsimony is a matter of degree. If Supervenience produces any 'amount' of gain in ontological parsimony, this will speak in favour of Supervenience. Of course it would be ideal if any loss in ontological parsimony due to the non-separability of comparativism would not outweigh the gain due to Supervenience, but when comparing regularity comparativism to comparativism simpliciter any 
Copyright Philosophy of Science 2017

Preprint (not copyedited or formatted)

Please use DOI when citing or quoting

degree of gain in ontological parsimony would provide a justification for Supervenience by itself.

Why then would Supervenience be more ontologically parsimonious than governing law views such as primitivism which take laws to be irreducible nomic facts over and above the occurrent facts (Maudlin 2007a, Ch.1)? Presumably because the Humean helps herself only to the mosaic, and does not require any additional nomic facts over and above the occurrent facts. But that is simply the wrong comparison. The governing law camp helps themselves, fundamentally, not to the full 4D mosaic, but merely to some initial conditions (i.e. a 3D mosaic) ${ }^{8}$ plus fundamental laws (or dispositions). The remaining dimension of the Humean mosaic, eternally far towards the past and into the future, supervenes on those initial conditions and the laws. Moreover, the governing law camp can help themselves to a small set of 'timeless' mass ratios - they hold at the initial time, and since the law does not evolve them, they hold at all times - whereas the Humean needs to 'buy into' instantaneous mass ratios for every instant of the 4D mosaic (and these mass ratios need to always be the same, without any explanation given).

Where does this leave us? Now it is clear that the Humean's fundamental ontology is not simply a subset of the ontology of the opposing camp, but instead a different set of 'things', it becomes difficult to say much about their relative ontological parsimony. I do not intend to settle this debate here. Instead I will end this section by pointing out that, even if the criterion of ontological parsimony would favour a Humean account of laws and regularity comparativism, in the next section we will see that such a principle brings with it a whole other threat to comparativism: eliminativism. Regularity comparativism finds itself in dangerous waters between the non-separable Scylla of Humean Empiricism (i.e. the dislike of necessary connections) and the eliminativist Charybdis of ontological parsimony.

\section{Stopping criterion \& Eliminativism}

A final objection against the regularity protocol is that it is "too easy" (Huggett 2006, 70). If the regularity approach succeeds in reducing inertial frames and absolute masses, why not use it to reduce any other notion as well (Arntzenius 2012)? Pooley, for instance, worries that the approach could be used to reduce even the temporal metric and the Leibnizian relations $(2013,567)$ ? It seems we need a non-ad-hoc criterion for determining how much stuff we can heave over from the supervenience base to the supervenient level.

But in the case of regularity relationalism it is not really clear why this is worrisome. As long as purging the supervenience base still produces the correct laws, is that not a good

\footnotetext{
${ }^{8}$ The issue remains of deciding which time is supposed to be the fundamental initial time (Casey McCoy, personal communication).
} 
Copyright Philosophy of Science 2017

Preprint (not copyedited or formatted)

Please use DOI when citing or quoting

thing? Especially Humean Empiricists should be happy to give up as many ontologically fundamental notions as possible, motivated by ontological parsimony.

In fact, Stevens succesfully outlines how one might reduce metrical notions - as Pooley mentioned - in the Special Relativistic case, by reducing them to a mosaic consisting only of topological relations (2014). Pooley even discusses such a project a bit later in the same paper $(2013,6.3 .2)$. He does correctly warn us that we might not be able to throw away everything from the supervenience base, because at some point we would not expect the regularity approach to generate the Newtonian laws anymore (but presumably some 'simpler' laws) (2013, fn.88). But from this it does not follow that we need some independent criterion to ensure that we stop throwing away stuff from the supervenience base at exactly that point; it is the regularity approach itself that tells us that it will break down at that point and we should thus not purge the supervenience base any further.

The case of regularity comparativism is importantly different. Empiricism, or more generally the desideratum of ontological parsimony, should welcome the opportunity of not only reducing absolute masses, but also mass ratios. In other words, regularity eliminativism - where the mosaic is purged of any mass properties - should be preferable over regularity comparativism. (In fact, this view was suggested for independent reasons by Hall (2012), and praised for its virtues by Esfeld et al. ${ }^{9}$ ) But this would imply that, by adopting the regularity approach, the comparativist has thrown away the massive baby with the bath water. Given that the regularity comparativist had already recognised the empirical meaningfulness of absolute masses, as encoded in the different particle trajectories in the mosaic, regularity eliminativism would combine with weak absolutism - the corresponding picture of the world would be a purely spatio-temporal mosaic which grounds the masses which in turn ground the mass ratios. Comparativism has overshot its goal.

For the comparativist it then does become important to provide an independent criterion for preventing the reduction of mass beyond the absolute masses. Elsewhere (2016, 2017) I do in fact provide an argument against eliminativism simpliciter. However, as is made clear there, it is exactly the regularity version of comparativism which cannot help itself to that argument. I consider whether initial conditions and laws which are purged from any notion of mass by replacing it with spatio-temporal quantities could still solve the corresponding set of initial value problems. The answer is negative. But along the way I discuss a less restricted attempt at reducing mass to spatio-temporal quantities by Narlikar (1939). Narlikar argues that, if we help ourselves to spatio-temporal quantities at a certain number of distinct instants - something we were not allowed to help ourselves to in the previous setting - we can reduce the mass ratios. And this should not be surprising. We only ever successfully discovered Newton's laws and the law of universal gravitation

\footnotetext{
${ }^{9}$ See Loewer (1996), Esfeld et al. (Esfeld 2014; Esfeld, Lazarovici, Lam, and Hubert 2015; Esfeld, Deckert, and Oldofredi 2015; Esfeld and Deckert 2016; Vassallo, Deckert, and Esfeld 2017) and Hubert (2015).
} 
Copyright Philosophy of Science 2017

Preprint (not copyedited or formatted)

Please use DOI when citing or quoting

from a set of data consisting of (a subset of) the eliminativist mosaic. The eliminativist project in Narlikar's sense must be true somehow.

In other words, it is unclear why any Humean Empiricist was committed to (Quantity) Primitivism - either about absolute masses or mass ratios - in the first place. Regularity eliminativism seems the most harmonious version of the regularity approach anyway. So much the worse for comparativism.

\section{Conclusion}

This paper applied Huggett's regularity approach - a liberalised version of the Mill-RamseyLewis Best Systems Account within the framework of (Humean) Supervenience - to comparativism about mass within Newtonian Gravity. It seems that the regularity approach indeed succeeds in its aim, once that is correctly interpreted as recovering the product of Newton's constant and the absolute mass from a comparativist Humean mosaic. It nevertheless clashes with the Separability clause of the Humean framework. An even more important reason for concluding that regularity comparativism is untenable is that once we employ the regularity approach in order to reduce away absolute masses, there is no reason not to also reduce the mass ratios. The regularity approach to comparativism throws away the massive baby with the bath water. 
Copyright Philosophy of Science 2017

Preprint (not copyedited or formatted)

Please use DOI when citing or quoting

\section{References}

Albert, D. (1996). Elementary quantum metaphysics. In J. T. Cushing, A. Fine, and S. Goldstein (Eds.), Bohmian Mechanics and Quantum Theory: An Appraisal, pp. 27784. Kluwer Academic Publishers.

Arntzenius, F. (2012). Space, Time, $\&$ Stuff. Oxford University Press.

Baker, D. J. (Manuscript, May 2013). Some consequences of physics for the comparative metaphysics of quantity.

Chen, E. K. (Manuscript, Jan. 2016). Our fundamental physical space: An essay on the metaphysics of the wave function.

Dasgupta, S. (2013). Absolutism vs comparativism about quantity. In K. Bennett and D. W. Zimmerman (Eds.), Oxford Studies in Metaphysics, Volume 8, pp. 105-47. Oxford University Press.

Dewar, N. (Manuscript, April 2015). What the humean cannot say about entanglement. http://philsci-archive.pitt.edu/12046/.

Earman, J. (1984). Laws of nature: The empiricist challenge. In R. J. Bogdan (Ed.), D.M. Armstrong, Volume 4 of the series 'Profiles', pp. 191-223. Dordrecht: D. Reidel Publishing Company.

Esfeld, M. (2014). Quantum humeanism, or: Physicalsm without properties. The Philosophical Quarterly 64(256), 453-70.

Esfeld, M. and D.-A. Deckert (Manuscript, 3 Feb. 2016). What there is: A minimalist ontology of the natural world.

Esfeld, M., D.-A. Deckert, and A. Oldofredi (Manuscript, 14 Oct. 2015). What is matter? the fundamental ontology of atomism and structural realism. arXiv:1510.03719v1.

Esfeld, M., D. Lazarovici, V. Lam, and M. Hubert (2015). The physics and metaphysics of primitive stuff. The British Journal for the Philosophy of Science Advance Access published July 72015.

Fraassen, B. V. (1970). An Introduction to the Philosophy of Time and Space. New York: Columbia University Press.

Hall, N. (Manuscript, 2012). Humean reductionism about laws of nature. http: //philpapers.org/archive/HALHRA.pdf. 
Copyright Philosophy of Science 2017

Preprint (not copyedited or formatted)

Please use DOI when citing or quoting

Hubert, M. (Manuscript, 10 December 2015). Quantity of matter or intrinsic property: Why mass cannot be both. http://philsci-archive.pitt.edu/11806/.

Huggett, N. (2006). The regularity account of relational spacetime. Mind 115(457), 41-73.

Hume, D. (Reprint 1894/1777). An Enquiry Concerning the Human Understanding. Oxford Clarendon Press.

Lewis, D. (1973). Counterfactuals. Cambridge: Harvard University Press.

Lewis, D. (1986). Philosophical Papers, Volume ii. Oxford University Press.

Loewer, B. (1996). Humean supervenience. Philosophical Topics 24(1), 101-27.

Martens, N. C. M. (Manuscript, Jan. 2017). Against comparativism about mass in newtonian gravity - a case study in the metaphysics of scale. DPhil Thesis, Magdalen College, University of Oxford.

Martens, N. C. M. (Manuscript, July 2016). Against reducing newtonian mass to kinematical quantities. http://philsci-archive.pitt.edu/12309/.

Maudlin, T. (2007a). The Metaphysics within Physics. Oxford University Press.

Maudlin, T. (2007b). Why be humean? In Maudlin (2007a), pp. 50-77.

Narlikar, V. (1939). The concept and determination of mass in newtonian mechanics. The London, Edinburgh, and Dublin Philosophical Magazine and Journal of Science: Series 7 27:180, 33-36.

Pooley, O. (2013). Substantivalist and relationalist approaches to spacetime. In R. Batterman (Ed.), The Oxford Handbook of Philosophy of Physics, pp. 522-86. Oxford University Press.

Stevens, S. (2014). The Dynamical Approach to Relativity as a Form of Regularity Relationalism. Ph. D. thesis, Exeter College, University of Oxford.

Vassallo, A., D.-A. Deckert, and M. Esfeld (Forthcoming, 2017). Relationalism about mechanics based on a minimalist ontology of matter. European Journal for Philosophy of Science. 